\title{
The Effects of COVID-19 Pandemic on the Follow-Up and Treatment Process of Gynecological Cancers and Breast Cancer
}

\author{
(1) Reyhan AYDIN DOĞAN,' (1) Neriman GÜDÜCÜ2 \\ 'Department of Midwifery, Karabuk University Faculty of Health Sciences, Karabuk-Turkey \\ ${ }^{2}$ Department of Midwifery, Istanbul University Cerrahpaşa Faculty of Health Sciences, Istanbul, Turkey
}

\begin{abstract}
SUMMARY
The present review was written to describe the effects of the COVID-19 pandemic on followup and treatment process of gynecological cancers and breast cancer in accordance with current literature, guidelines from national and international cancer associations. Recommendations were provided based on the consensus conference model. In the follow-up and treatment process of gynecological cancers and breast cancer, there are many factors, such as the clinical course of patient, the possibility of an emergency of the patient, the stage of the cancer and the current status of the chemotherapies. Although opinions in all types of cancer differ among themselves, general beliefs are postponement of non-urgent surgical operations, re-evaluation of chemotherapy processes, postponing appointments for new nonurgent diagnoses, reducing the length of hospital stay of patients in emergency procedures, conducting consultations by telemedicine or telephone, treatment of patients with COVID-19 suspicions after 15 days, planning biopsies or surgeries according to the staging status of cancers and planning conferences as video conferences in cases that require a multidisciplinary approach. Patients diagnosed with cancer during the COVID-19 pandemic should not be lost, followup and treatment should be continued without disruption, patients should be remembered to be in the high-risk group in this process and necessary hygiene measures should be taken in both follow-up and treatment concerning risks and COVID-19 pandemics.
\end{abstract}

Keywords: COVID-19 and breast cancer; COVID-19 and cancer management; COVID-19 and gynecological cancers. Copyright $\odot$ 2021, Turkish Society for Radiation Oncology

\section{Introduction}

The new type coronavirus COVID-19 announced as a pandemic by the World Health Organization [WHO] on March 11,2020, began to spread rapidly all over the world.[1-4] This process lead to a public health problem all over the world and in Turkey.[2,5] During the pandemic, hospitalization rates, the number of patients requiring intensive care support, and the rates of infec- tion with the COVID-19 virus continued to increase. $[6,7]$ In this process, the need for reorganization of hospitals and intensive care units, the routine patient control process, the follow-up of chronic diseases, the treatment process of diseases that require regular care, and new arrangements for emergencies emerged.[8] Many people in the world were affected by this process; however, it was determined that $20 \%$ of those who died from COVID-19 also had cancer.[7,9] International Agency for Research on Cancer also reported that peo- 
ple with cancer are in the high-risk group during the COVID-19 process. $[9,10]$

After the spread of COVID-19 cases, cancer research centers and institutes made consensus on the follow-up, treatment and prevention of pandemic cancer patients in this process. [8] At the same time, medical oncologists also published guidelines on this treatment process. $[9,11]$ In addition, the European Society for Medical Oncology [ESMO] created useful links and question-answer platforms on the webpages for cancer patients.[11] To inform cancer patients, the Turkish Society of Medical Oncology made recommendations for cancer patients and their relatives on its webpages in line with the literature.[12,13]

In the investigation of cancer and COVID-19 incidence, scientists emphasized that in the first outbreak, the number of cancer patients who died in Wuhan University Zhongnan Hospital was 12 and this number had $0.37 \%$ of the general population. $[6,14]$ It was emphasized that the cancer type most affected by COVID-19 was lung cancer, while the incidence of people aged 60 and over was 4.3\%.[6,14] During the pandemic, it was observed that 28 cancer cases came to Wuhan University Zhongnan Hospital and 10 [35.7\%] of these cases were in the fourth stage.[14] The causes of death of these patients were mostly lung failure, and the other causes were a septic shock, myocardial infarction and pulmonary embolism, respectively. $[6,14]$

In a report published by the Turkey Cancer Statistics Center in 2018, cancer rates in Turkey are above the world incidence in men; the most common type of cancer in men is lung cancer, while women have breast cancer.[15] In this context, it was seen that cancer patients in Turkey are at great risk in the COVID-19 epidemic, just as the whole world seems to be greatly at risk. The present review was written to describe the effects of the COVID-19 pandemic on the follow-up and treatment process of gynecologic cancers and breast cancer in line with current literature, national and international cancer associations' guidelines.

\section{Materials and Methods}

Recommendations based on the consensus conference model were made.[7,10,11,13,16-18]

\section{Follow-up, Treatment and Operation Processes of Cancer Patients during COVID-19 Pandemic}

Many factors, such as age, chronic disease presence, previous cancers, weight, psychology, smoking, alcohol use, diet and sexually transmitted diseases, are effective in the process of cancer follow-up and treatment.
[19] A new risk factor has emerged for cancer patients after the initiation of the COVID-19 pandemic. In this process, cancer patients are more susceptible to infections than healthy subjects due to the suppression of their immune systems, attracting them to the focus of the pandemic.[20] Simultaneously, the high age group of cancer patients was effective in this process.[15,21] Cancer research centers and hospitals have determined changes and protocols in treatment and follow-up processes according to cancer types, duration and stage as the pandemic progress.[11,12,21]

The information about gynecological cancers and breast cancer below includes cancer follow-up and treatment information in the COVID-19 process of people who are experts in their field and are involved in cancer field studies in various regions of the world.

\section{Outpatient Treatment Process in Gynecological Cancers [Outpatient Patients]}

During the COVID-19 process, gynecological cancers were restricted in outpatient treatments and examinations, as in other types of cancer.[22] Ramirez et al.[22] listed the outpatient and outpatient follow-up of gynecological cancers as follows:

- Restricting the outpatient follow-up of patients diagnosed with new cancer,

- To reduce exposure with COVID-19, midwives, nurses and physicians and other service providers,

- Limiting the individuals accompanying patients in the cancer treatment process to one person and regular control of this accompanying person against the epidemic,

- As the conditions of the institutions permit, until the end of the epidemic, patient evaluations and consultations are performed on a web-based, telephone or online basis.

- Evaluating the reviews of the cases where the results are risky and need additional views and consultations, in a web-based environment

- Postponement of non-symptom cases and cases where there are no problem in the final examination, radiological imaging, blood tests or interventions.[22]

\section{The Process of Inpatient Treatment Patients in Gyne- cological Cancers}

COVID-19 pandemic has a significant effect on people with chronic disease; oncologists have decided to postpone their treatment since the immune systems of patients in the cancer treatment process are adversely 
affected.[9,23] Akladios et al.[23] stated the planning suggestions for surgical and chemotherapy treatments in gynecological cancers as follows:

- In addition to surgical treatment in patients with pelvic gynecologic cancer, all other alternatives should be considered,

- Especially surgical operations should be treated with the risks of benefits and losses,

- Considering complications after the operation, intensive care bed occupation should also be considered,

- In case of COVID-19 positive patient, treatment should be postponed for 15 days,

- Every patient who comes to the clinic for mandatory procedures must wear a mask,

- Radiation therapy for localized cancers, cancer that has deteriorated too much to be cured by considering age, life span and general condition should be planned for treatment,

- Considering the incubation period of the pandemic, each patient should be considered COVID-19 positive and measures should be taken,

- Materials in clinical studies with patients with cancer should be stored in accordance with the storage conditions,

- Patient evaluations should be recorded by video or teleconference,

- The location, value, and imaging test results of lymph node staging surgeries, such as cervical cancer should be reviewed according to the stage,

- Oncological consultations should be postponed for two months after treatment,

- If the institution's condition is favorable, teleconsultation should be addressed, but in gynecological cancer types requiring examination such as cervical and endometrial cancers, the follow-up should be postponed for two months. [23]

- SGO [Society of Gynecological Oncology] is of the opinion that delay is unacceptable from a psychological standpoint, except for delays of up to 3-8 weeks for oncological specific cancer cases. SGO argues that it would be appropriate to direct semiemergency patients to regional colleagues if it is possible and safe to continue surgical treatment.

\section{Management of Cervical Cancer during COVID-19 Pandemic}

Cervical cancer follow-up and treatment management varies according to the location and stage of cancer. $[16,22]$
According to the American Society for Colposcopy and Cervical Pathology Association [ASCCP], assessments of women at low risk of cervical screening test results in pre-invasive cases should be delayed by $6-12$ months. American Society for Colposcopy and Cervical Pathology recommends conducting a diagnostic evaluation within three months for women at high risk for diagnostic tests.[16,22,23]

ASCCP suggests that in early-stage cervical cancers, clinics where oncological surgery is permitted, the surgical operation should be planned and also the next treatment steps should be planned by radiological imaging of the disease. [16,22,23]

In advanced cervical cancers, the need to reduce the number of hospital admissions is emphasized. [16,22-24] American Brachytherapy Society has stated that patients should continue brachytherapy, but doses should be scheduled weekly, not daily, and should be administered with telemedicine in daily visit situations.[22,24]

\section{Management of Ovarian Cancer during COVID-19 Pandemic}

It is emphasized that the ovarian cancer treatment process should be planned according to the clinical condition of the patient and the stage of cancer.[22,23]

It is recommended to evaluate people with age, familial predisposition or physical examination on suspicion of ovarian cancer by considering the risks of malignancy and blood tests.[22,23]

It is suggested to perform a biopsy for patients with advanced-stage diagnosis to confirm the diagnosis and continue three or four cycles of chemotherapy treatment until the results are obtained, and then plan the surgical procedure according to the suitability of the surgery.[22,23] It is recommended to extend the treatment of patients who previously started chemotherapy treatment for up to six months instead of three months, and then plan the surgical operation.[22,23] At the same time, either histological analysis or complete surgery is recommended for those with suspected ovarian mass.[22,23]

It is proposed to introduce travel restrictions for patients with travel necessity for the treatment of ovarian cancer, to conduct treatment arrangements with oncologists in their region during the pandemic process and to communicate via telemedicine.[22] In cases where ovarian cancer exists and the disease progresses, it is advised to evaluate the clinical conditions for additional treatment and to decide on the benefit-harm potential of the agents used.[22] 


\section{Management of Endometrial Cancer during COVID-19 Pandemic}

Endometrial cancer treatment is planned according to its stages as in other types of cancer.[23] In endometrial cancers, which are suspicious in post-menopausal bleeding or metroragie cases, it is recommended to use pipelines and avoid diagnostic hysteroscopy.[23] It is mentioned that the definitive treatment should be planned according to the degree of cancer suspicion, the patient's age, genetic status and other risk factors.[23]

Non-surgical, non-invasive methods and operation, additionally postponing the operation by a month or two are recommended for early-stage and low-risk endometrial cancers. [22,23]

Hysterectomy and bilateral salpingo-oophorectomy are recommended for the treatment of second or third stage, high-risk endometrial cancers.[22] Laparotomy or laparoscopy status for surgical operations is controversial.[22] Biopsy is strongly advised to diagnose progress and confirm the follow-up process for advanced endometrial cancers. [22,23]

\section{Management of Vulva/Vagina Cancers during COVID-19 Pandemic}

Since the most important and generally presented standard treatment in the treatment of vulva cancer is the surgical treatment method, it is endorsed that this treatment should not be changed.[23] It is reported that the treatment process can be delayed for a few weeks in the case of tumor tissue due to the high incidence of vulva cancer and generally affects the elderly. [23] It is recommended that the follow-up process continues at home after the operation. The application of chemotherapy after vulva cancer is controversial.[23] In the management of vagina cancer and COVID-19, it is recommended that the follow-up treatment process is reviewed and planned according to stage of the cancer and the extent of the spread to the lymph nodes due to the need for radiotherapy, brachytherapy and chemotherapy.[23]

\section{Management of Trophoblastic Tumors during COVID-19 Pandemic}

Trophoblastic tumors are considered metastatic for all patients and the treatment process should not be delayed.[23] Treatment of these tumors should be planned according to staging and risk conditions.[23] Home treatments are suggested for low-risk cancers. [23] It is recommended to start using multiple drug applications without delaying therapy for patients in the high-risk group.[23] Ultrasound-controlled curettage is approved and International Federation of Gynecology and Obstetrics [FIGO] suggests that the post-procedure treatment protocol can be maintained at home with the use of methotrexate for the treatment of molhidatiform pregnancies.[17,23]

\section{Management of Breast Cancer during COVID-19 Pandemic}

Breast cancer is the most common cancer among women in the world.[25] One in four women has been diagnosed with breast cancer in Turkey.[15] Many current treatment methods, such as hormone therapy, chemotherapy or breast reduction, are considered for the treatment of breast cancer.[25] Furthermore, as in other types of cancer, the decision should be made by considering the status of inpatient services, the status of intensive care units and the patient's intensive care after treatment.[25]

Follow-up and treatment of breast cancer should be planned according to the clinical course of the patient, and it is thought that postponing surgical operations to a later date does not affect the survival rates of the patients.[25] However, there is no definite judgment about postponing surgical operations after the COVID-19 pandemic has completely ended, and this result is controversial.[25]

The scientific board of the Magee-Womens Breast Cancer Program, one of the most intense breast cancer centers in America, published the following recommendations regarding breast cancer follow-up and treatment $[18,25,26]$ :

- While performing breast cancer screenings, non-emergency routine screenings will be postponed to a later date,

- Biopsy and imaging procedures requiring immediate diagnosis will continue in the same way,

- For minor interventions, such as seed placement [or wire], procedures will be planned for patients coming from outside the city on the same day,

- For pathologies, time planning will be made in biopsies, it will be predicted that there will be a delay in the results reports, frozen materials taken during the operation will either be made in the operating room or the result will be reported by phone,

- Surgical consultations will be discussed face-toface with patients and other routine patients in a virtual interview. Meeting dates will be postponed for those who will not be able to provide virtual interviews,

- An emergency treatment method will be planned 
for patients whose surgical treatments cannot be postponed. In addition, alternative medical interventions, such as hormone therapy, systemic chemotherapy, taking recommendations from medical oncologists, the continuation of patients who have completed chemotherapy, systemic treatment, high-risk or benign surgeries, and operations that reduce risk will be offered,

- Making observations with telemedicine, continuing infusions and annual injections and following metastatic cancers in accordance with the protocols for medical oncology,

- Planning the operations according to the general condition of the patients planned for breast plastic surgery and postponing the non-urgent reconstruction surgeries,

- Offering consultations with telemedicine for radiation oncology and keeping most patients' treatment the same,

- Drawing blood on the same day and examination of emergency patients who will be seen directly in genetic screenings, providing consultation with telemedicine to non-emergency patients and planning face to face interviews with emergency patients only,

- Closing of biospecimen laboratories for researches and keeping clinical research open,

- Multidisciplinary meetings are proposed as video conferences.[25-27]

\section{Result}

In the follow-up and treatment process of gynecological cancers and breast cancer, there are many factors, such as the clinical course of the patient, whether there is an emergency, the stage of cancer, and the current state of chemotherapy. The information in the present review is based on expert opinions determined for each type of cancer.[7,10,12,13,16-18] It is thought that it will be healthier for patients to plan their own course in line with the opinions of national and international cancer programs. It should be noted that international cancer programs are regularly updated. Each institution should maintain its social distances in line with the recommendations of the World Health Organization and disease control and prevention centers and should always pay attention to national and international hygiene recommendations.
Peer-review: Externally peer-reviewed.

Conflict of Interest: I have no conflict of interest.

Financial Support: I have no financial support.

\section{References}

1. Cafarotti S, Patella M. Lung Cancer Surgical Management During the Outbreak of Coronavirus Disease 2019. J Thorac Oncol 2020;15(6):e81

2. Calabrò L, Peters S, Soria JC, Di Giacomo AM, Barlesi F, Covre A, et al. Challenges in lung cancer therapy during the COVID-19 pandemic. Lancet Respir Med 2020;8(6):542-4.

3. Chen N, Zhou M, Dong X, Qu J, Gong F, Han Y, et al. Epidemiological and clinical characteristics of 99 cases of 2019 novel coronavirus pneumonia in Wuhan, China: a descriptive study. Lancet 2020;395(10223):507-13.

4. Shi H, Han X, Jiang N, Cao Y, Alwalid O, Gu J, et al. Radiological findings from 81 patients with COVID-19 pneumonia in Wuhan, China: a descriptive study. Lancet Infect Dis 2020;20(4):425-34.

5. Ministry of Health. Türkiye Republic Ministry Of Health Corona Table [Internet]. Available at: https:// covid19.saglik.gov.tr. Accessed Apr 01, 2020.

6. Huang C, Wang Y, Li X, Ren L, Zhao J, Hu Y, et al. Clinical features of patients infected with 2019 novel coronavirus in Wuhan, China. Lancet 2020;395(10223):497506.

7. World Health Organization. Novel coronavirus [COVID-19] situation [Internet]. Available at: https:// www.who.int/emergencies/diseases/novel-coronavirus-2019. Accessed Apr 16, 2020.

8. Emanuel EJ, Persad G, Upshur R, Thome B, Parker M, Glickman A, et al. Fair Allocation of Scarce Medical Resources in the Time of Covid-19. N Engl J Med 2020;382(21):2049-55.

9. Burki TK. Cancer guidelines during the COVID-19 pandemic. Lancet Oncol 2020;21(5):629-30.

10. The Lancet Oncology. COVID-19: global consequences for oncology. Lancet Oncol 2020;21(4):467.

11.ESMO. COVID-19 and Canser Manage [Internet]. Available at: https://www.esmo.org/covid-19-andcancer/supporting-oncology-professionals. Accessed Apr 15, 2020.

12. Turkish Society of Medical Oncology. Cancer patients and their relatives [Internet]. Available at: https:// www.kanser.org/saglik/toplum/style/Covid19_hasta_ onerileri.pdf. Accessed Apr 1, 2020.

13. Sümbül AT, Yalçın Ş, Özet A, Ünal S, Dizdar Ö, Akbulut $\mathrm{H}$, et al. Turkish medical oncology society 
covid-19 pandemic advisory board recommendations for cancer patients and medical oncologist. J Oncol Sci 2020;1(6):1-4.

14. Sidaway P. COVID-19 and cancer: what we know so far. Nat Rev Clin Oncol 2020;17(6):336.

15. Public Health Authority of The Republic of Turkey. Turkish Cancer Statistics 2015. Available at: https:// hsgm.saglik.gov.tr/depo/birimler/kanser-db/istatistik/ Trkiye_Kanser_statistikleri_2015.pdf. Accessed Nov 19, 2020.

16. ASCCP. ASCCP Interim Guidance for Timing of Diagnostic and Treatment Procedures for Patients with Abnormal Cervical Screening Tests [Internet]. Available at: https://www.asccp.org/covid-19. Accessed Apr 1,2020 .

17. FIGO. COVID-19 \& Management of Gynecological Cancers. International Federation of Gynecology and Obstetrics. Available at: https://www.figo.org/covid19-management-gynecological-cancers. Accessed Nov 19, 2020.

18. UPMC. COVID-19: The Latest Information from UPMC [Internet]. Available at: https://www.upmc. com/coronavirus?sc_camp=6EA251EE93FD44D58D 9346B41197F470. Accessed Apr 24, 2020.

19. Stein CJ, Colditz GA. Modifiable risk factors for cancer. Br J Cancer 2004;90(2):299-303.

20. Dowdy S, Fader AN. Surgical Considerations for Gynecologic Oncologists During the COVID-19 Pandemic. Society of Gynecologic Oncology 2020;1-4.

21. Lambertini M, Toss A, Passaro A, Criscitiello C, Cremolini $\mathrm{C}$, Cardone $\mathrm{C}$, et al. Cancer care during the spread of coronavirus disease 2019 (COVID-19) in Italy: young oncologists' perspective. ESMO Open 2020;5(2):e000759.
22. Ramirez PT, Chiva L, Eriksson AGZ, Frumovitz M, Fagotti A, Gonzalez Martin A, et al. COVID-19 Global Pandemic: Options for Management of Gynecologic Cancers. Int J Gynecol Cancer 2020;30(5):561-3.

23. Akladios C, Azais H, Ballester M, Bendifallah S, Bolze PA, Bourdel N, et al. Guidelines for surgical management of gynaecological cancer during pandemic COVID-19 period - FRANCOGYN group for the CNGOF. [Article in French]. Gynecol Obstet Fertil Senol 2020;48(5):444-7.

24. ABS Statement on Coronavirus. Available at: https:// www.americanbrachytherapy.org/about-abs/absnews/abs-statement-on-coronavirus/. Accessed Apr 23, 2020 .

25. Soran A, Gimbel M, Diego E. Breast Cancer Diagnosis, Treatment and Follow-Up During COVID-19 Pandemic. Eur J Breast Health 2020;16(2):86-8.

26. Gligorov J, Bachelot T, Pierga JY, Antoine EC, Balleyguier C, Barranger E, et al. COVID-19 and people followed for breast cancer: French guidelines for clinical practice of Nice-St Paul de Vence, in collaboration with the Collège Nationale des Gynécologues et Obstétriciens Français (CNGOF), the Société d'Imagerie de la Femme (SIFEM), the Société Française de Chirurgie Oncologique (SFCO), the Société Française de Sénologie et Pathologie Mammaire (SFSPM) and the French Breast Cancer IntergroupUNICANCER (UCBG). [Article in French]. Bull Cancer 2020;107(5):528-37.

27. Braunstein LZ, Gillespie EF, Hong L, Xu A, Bakhoum SF, Cuaron J, et al. Breast Radiation Therapy Under COVID-19 Pandemic Resource Constraints-Approaches to Defer or Shorten Treatment From a Comprehensive Cancer Center in the United States. Adv Radiat Oncol 2020;5(4):582-8. 